\title{
Guidelines for correlation coefficient threshold settings in metabolite correlation networks exemplified on a potato association panel
}

\author{
David Toubiana and Helena Maruenda (1)
}

${ }^{*}$ Correspondence: hmaruen@pucp.edu.pe Departamento de Ciencias - Química, Centro de Espectroscopia de Resonancia Magnética Nuclear (CERMN), Pontificia Universidad Católica del Perú, Av. Universitaria 1801, Lima 32, Lima, Peru

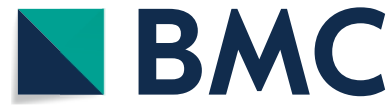

(C) The Author(s) 2021. Open Access This article is licensed under a Creative Commons Attribution 4.0 International License, which permits use, sharing, adaptation, distribution and reproduction in any medium or format, as long as you give appropriate credit to the original author(s) and the source, provide a link to the Creative Commons licence, and indicate if changes were made. The images or other third party material in this article are included in the article's Creative Commons licence, unless indicated otherwise in a credit line to the material. If material is not included in the article's Creative Commons licence and your intended use is not permitted by statutory regulation or exceeds the permitted use, you will need to obtain permission directly from the copyright holder. To view a copy of this licence, visit http:// creativecommons.org/licenses/by/4.0/. The Creative Commons Public Domain Dedication waiver (http://creativecommons.org/publi cdomain/zero/1.0/) applies to the data made available in this article, unless otherwise stated in a credit line to the data.
Keywords: Metabolite correlation network, Threshold settings, Correlation coefficient, Pearson correlation, Potato association panel, Metabolism, Mouse heart metabolism 


\section{Background}

Correlation-based network analysis (CNA) has become an integral tool for studying the coordinated behavior of metabolite profiles in plant sciences. Metabolite correlation networks $(\mathrm{CN})$ are constructed by correlating each two pairs of metabolites across a set of different conditions or by exploiting the natural variability of mapping populations or collection of varieties, as they provide large sample size stabilizing the correlation and reducing the error rate, e.g. in a tomato introgression line mapping population [1, 2], a variety collection of sparkling wines [3], a diverse collection of Arabidopsis accessions [4], and a maize association panel [5]. The coordinated behavior of metabolites across diverse varieties gives insights into their genetic communalities. Each pairwise correlation is represented by a correlation-coefficient $r$ ranging from -1 to 1 . In addition, the significance of each correlation is evaluated by computing a $p$-value ranging from 0 to 1 . In a metabolite $\mathrm{CN}$, nodes represent metabolites and the edges between them represent the estimated correlation coefficients.

To construct metabolite CNs, threshold restrictions are applied to the correlation coefficients and their associated $p$-values to identify spurious correlations between metabolites. Subsequently, the non-significant correlations or edges, respectively, are removed from the network. Threshold settings for the associated $p$-values follow the rules of multiple hypotheses testing, such as a false discovery rate (FDR) [6]. However, guidelines for threshold settings of the correlation coefficient have not been well defined yet.

Ideally, edges between nodes in a $\mathrm{CN}$ reflect metabolic fluxes through a metabolic pathway. However, the relationship between metabolic pathways and the correlations between metabolites is not straight forward. Factors, such as short-term fluctuations caused by plant variability or internal noise may render into weak correlations. Systematic changes of the steady-state as well as aspects of cellular organization also need to be taken into account. Furthermore, the involvement of metabolites in multiple pathways and their extensive crosstalk within, makes it difficult to clearly affiliate metabolites to metabolic pathways in CNs. Thus, the correlation coefficient threshold cannot be universally set and must be instead adjusted to the system of study in order to extract meaningful biological data [7]. As a result, different studies have applied different selective threshold settings, e.g. Hu et al. constructed metabolite CNs for Osteoarthritis and control patients to identify significantly changing correlations between networks [8]. There the authors set a threshold for edges based on the $p$-value only. Via topological analysis of the difference network, they managed to highlight key metabolites that played an important role in governing the connectivity and information flow of the network. In [9] the authors used a moderate correlation coefficient threshold of 0.6 which enabled them to identify genes affecting free amino acids. In [10] a threshold of 0.7 was applied to highlight the differences between metabolite networks of fed and fasted mice. Yet again in [11] a rigorous correlation coefficient threshold of 0.8 was employed identifiying metabolic patterns for freezing tolerance in two Brachypodium Sylvaticum ecotypes. The selection of the correlation coefficient threshold, which allows meaningful biological interpretation, depends on the network topology rather than on the strength of the correlation coefficient itself. That being said, network properties associated with node connectivity alter (or better said stop altering) once a certain threshold has been reached. In other words, the selection of the correlation coefficient threshold depends on the 
distribution of the network's numbers of edges at varying $p$-values, similar to the idea of choosing a $p$-value threshold based on the $p$-value distribution in the Benjamini-Hochberg FDR multiple hypotheses testing correction [6].

We have recently profiled the tuber of a potato association panel that was grown under normal irrigation (NI) and recovery (REC) treatments for 42 metabolites and also measured a set of 45 morphological traits of the plant. [12]. For each treatment, one $\mathrm{CN}$ was constructed, where the correlation coefficient threshold for both CNs was set to Pearson's $|r| \geq 0.4$. In addition, the profiles were utilized for a genome wide association study (GWAS). Via the application of set theory to networks, a difference network was constructed, highlighting the difference set of the REC network over the NI network (REC $\not \subset \mathrm{NI})$. In this perspective, the correlation between the metabolite fumaric acid and the morphological trait plant vigor was shown to be specific to the REC network. Next, we analyzed the single nuclear polymorphisms associated with fumaric acid and identified a gene coding for a RING domain protein on chromosome 1 in the potato genome and a gene coding for a zinc finger protein ZAT2 on chromosome 4. It was demonstrated before that both genes are essential for plants to cope with abiotic stresses [13-18].

In the current study, we demonstrate that the estimated correlation coefficient threshold of Pearson's $|r| \geq 0.4$ was crucial for the establishment of a connection between fumaric acid and plant vigor, and by that for the identification of the aforementioned regulating genes. By in silico manipulations of the tuber $\mathrm{CN}$ and monitoring its connectivity between nodes, we define guidelines onto how to identify the proper correlation coefficient threshold for metabolite CNs. Last, we apply the proposed method on a mouse metabolite dataset to prove its efficacy on a dataset of different origin.

\section{Results}

Initial networks

We defined the NI and REC-CNs as weighted networks $G_{i}=\left(V_{i}, E_{i}, w\right)$, where $V_{i}$ was the set of nodes corresponding to metabolites and morphological traits found in the dataset of treatment $i, E$ was the set of edges between them, and edge weights $(w: E \rightarrow R)$ corresponded to the Pearson correlation coefficient. Thresholds for both networks were set to Pearson's $|r| \geq 0.4$ and a $q$-value $\leq 0.05$, removing spurious correlations. At these settings, the NI-CN had $\left|V_{N I}\right|=88$ nodes and $\left|E_{N I}\right|=438$ edges connecting them; the REC-CN was composed of $\left|V_{R E C}\right|=90$ nodes and $\left|E_{R E C}\right|=370$ edges. The connection between fumaric acid and plant vigor was present in the REC-CN but not in the NI-CN, as the corresponding correlation coefficients were computed with 0.458 and 0.013 , respectively.

\section{Determining the correlation coefficient by testing the number of edges}

As described above, the integration of a correlation into a $\mathrm{CN}$ depends on two threshold settings, namely the correlation coefficient and the associated $p$-value. In this and in other studies it was observed that the number of edges in a $\mathrm{CN}$ remains stable despite gradually increasing the $p$-value stringency settings until a certain correlation coefficient has been reached. In other words, the proposed method quantifies the number of edges that would be integrated into the $\mathrm{CN}$ dependent on the absolute correlation coefficient and its associated $p$-value. It does so in descending order at decrements of 0.1 of the 
correlation coefficient and ranging $p$-values of 0.05 to 0.01 . Once the number of edges starts dropping at a certain correlation coefficient, the threshold is set to this value. We tested for significant changes in edge number by estimating the confidence intervals (CI) at 95\% employing the modified Cox method [19] assuming non-normal distributions, such that:

$$
\begin{aligned}
& \text { decrement }:=\text { corcoef } * .1 \\
& \text { edge.number.changed }:=\text { FALSE } \\
& \text { p.values }:=[0.05,0.04,0.03,0.02,0.01] \\
& \text { while (edge.number.changed }==\text { FALSE) }\{ \\
& \text { edge.number }:=\text { correlation_network(corcoef, p.values) } \\
& \text { if (min(edge.number) }<\text { mean(edge.number) }- \text { error OR } \\
& \text { max (edge.number) }>\text { mean(edge.number }+ \text { error) } \\
& \text { then } \\
& \text { edge,number.changed }:=\text { TRUE } \\
& \text { corcoef }:=\text { corcoef }- \text { decrement }
\end{aligned}
$$$$
\text { if (min (edge.number) < mean (edge.number) - error OR }
$$

where the term corcoef corresponds to the initial correlation coefficient, edge.number. changed is a Boolean variable, edge.number is an array variable of function correlation network, computing the number of edges of different $\mathrm{CNs}$ at parameters corcoef and p.values, and the error term is defined as CI / 2.

To demonstrate this approach, we constructed a series of NI and REC networks at varying correlation coefficient and $p$-value settings and quantified the number of edges present (Fig. 1). The figure shows that the number of edges remained unchanged at varying $p$-values until the Pearson's correlation coefficient dropped to 0.2 in the NI-CN (Fig. 1a and b), suggesting that the correlation coefficient threshold ought to be located somewhere in-between 0.2 and 0.3 . At a correlation coefficient of 0.2 the edge number ranged between 923 and 948, the mean was estimated with 938.8, the upper limit of the 95\% CI was calculated with 951.34, the lower limit with 926.45. For the REC-CN a significant drop was registered at a correlation coefficient of 0.4, suggesting that the threshold was supposed to be located somewhere in-between 0.4 and 0.5 (Fig. 1c and d). The associated values were estimated with mean $=373.4$, the upper limit of the $\mathrm{CI}=376.66$, the lower limit of the $\mathrm{CI}=370.18$, the minimum $=370$, and the maximum $=376$.

\section{Fine-tuning computes the correlation coefficient thresholds with 0.23 and 0.41}

Next, we investigated CNs at different $r$-values in the range of 0.2 to 0.3 for the NI-CN and in the range of 0.4 to 0.5 for the REC-CN (Fig. 2). As before, the $r$ threshold value 

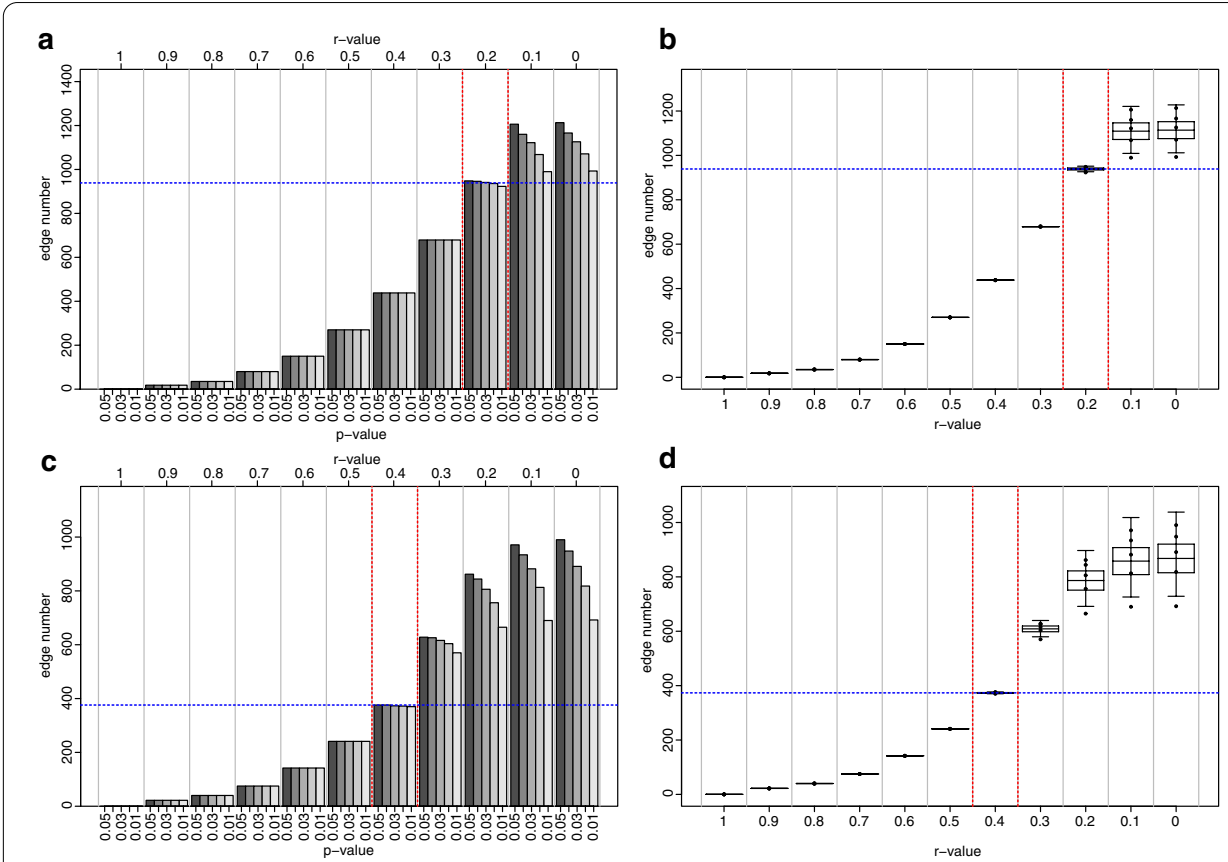

Fig. 1 Edge number distribution of $\mathrm{Nl}$ and REC networks, $r=1$ to 0 . Graphs on the left-hand side of the figure illustrate histograms of edge number in the NI and REC-CNs at different $r$-value to $p$-value combinations, at $r=1$ to 0 at decrements of 0.1 . Graphs on the right-hand side of the figure depict corresponding boxplots, where the centerlines represent the mean; box limits represent upper and lower standard error; whiskers represent $95 \%$ confidence intervals calculated by the modified Cox test. Grey vertical lines separate correlation coefficients, red dashed vertical lines represent proposed correlation coefficient threshold interval, blue horizontal lines represent the mean edge number at which threshold is set; a NI-CNs edge number histogram, b NI-CNs edge number boxplot, $\mathbf{c}$ REC-CN edge number histogram, $\mathbf{d}$ REC-CN edge number boxplot

was defined as the $r$-value when the minimum or maximum edge number was located outside the confines of the corresponding CI. This behavior occurred for the NI-CN at a correlation coefficient threshold Pearson's $|r| \geq 0.23$. Here, the edge number ranged between 850 and 853, the mean was calculated with 851.8, the CI lower limit was computed with 850.18 and the CI upper limit with 853.42 (Fig. 2). For the REC-CN this behavior was observed at Pearson's $|r| \geq 0.41$. At this $r$-value the edge number ranged between 354 and 359, the mean was computed with 357, the CI upper limit with 359.65 and the CI lower limit with 354.37. Consequently, the $r$-value threshold for the NI-CN was proposed with 0.23 and for the REC-CN with 0.41 .

\section{Bootstrapping analysis confirms correlation coefficient thresholds}

To verify the proposed correlation coefficient threshold settings, we employed bootstrapping with replacement; such that $100 \mathrm{NI}$ and REC-CNs were generated with $80 \%$ of the samples randomly selected, where one sample could be part of the sample subset more than once. Performing this analysis, we wanted to validate whether the estimated threshold was due to chance or indeed the result of the network topology at the proposed threshold even at a reduced set of samples (80\%). As before, the analysis was divided into two cycles. For the first cycle the edge numbers of all networks were 


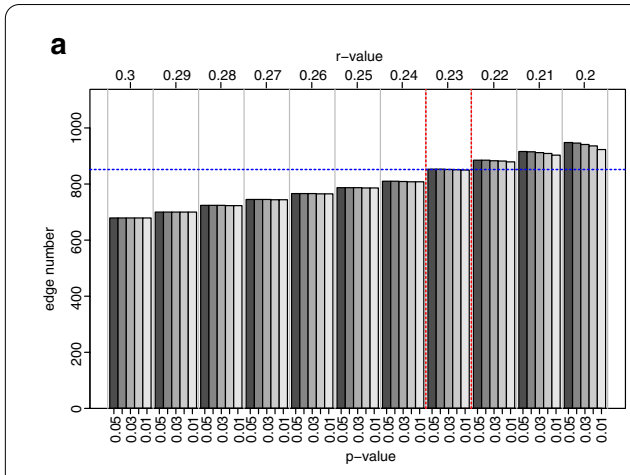

b

c
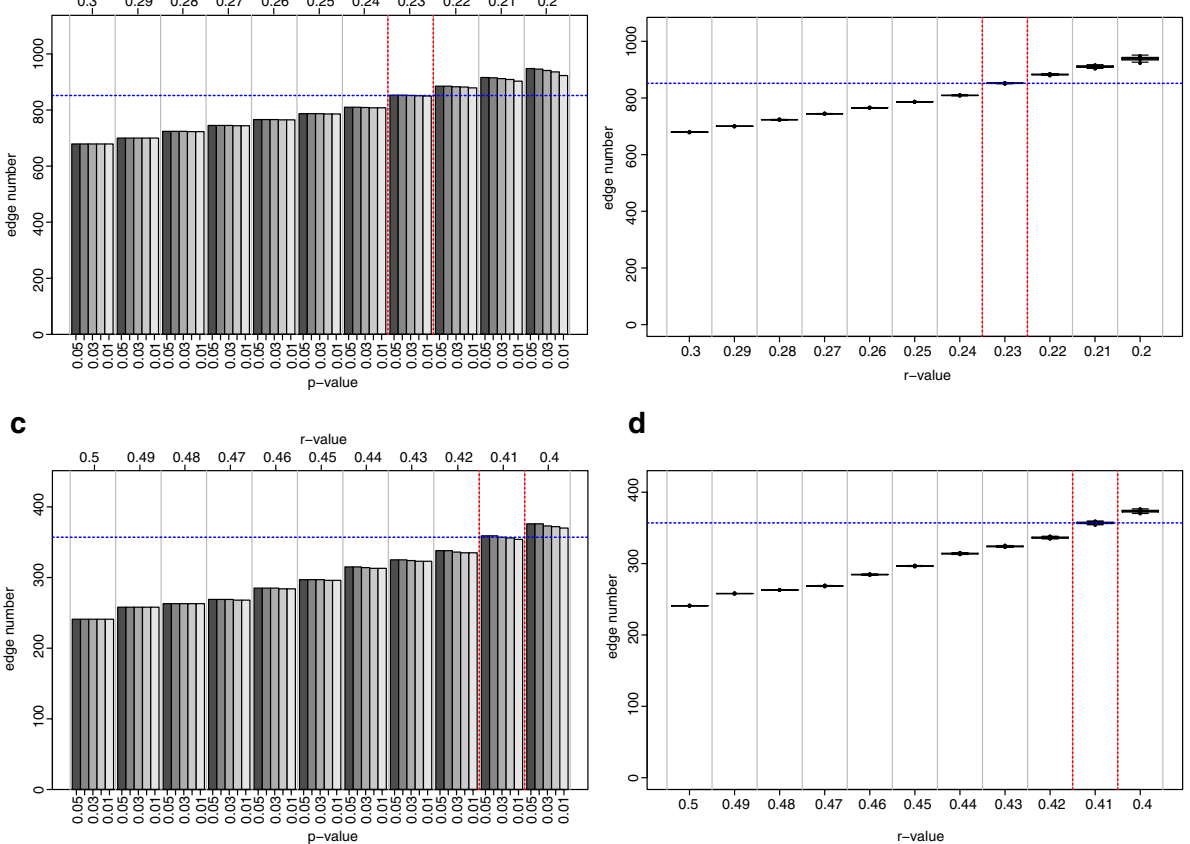

d

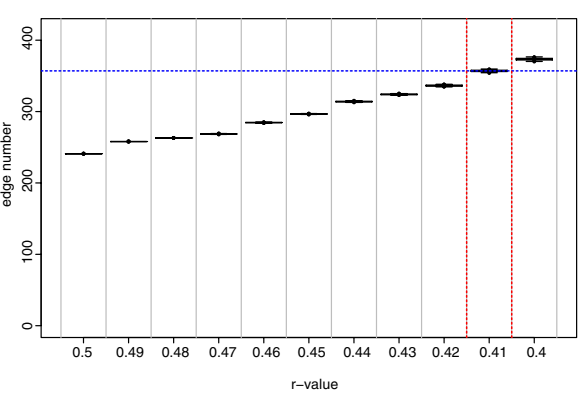

Fig. 2 Edge number distribution of $\mathrm{NI}$ and REC networks, $r=0.3$ to 0.2 and 0.5 to 0.4 . Graphs on the left-hand side of the figure illustrate histograms of edge number in the NI and REC-CNs at different $r$-value to $p$-value combinations, at $r=0.3$ to 0.2 and 0.5 to 0.4 at decrements of 0.01 , respectively. Graphs on the right-hand side of the figure depict corresponding boxplots, where the centerlines represent the mean; box limits represent upper and lower standard error; whiskers represent 95\% confidence intervals calculated by the modified Cox test. Grey vertical lines separate correlation coefficients, red dashed vertical lines represent proposed correlation coefficient threshold interval, blue horizontal lines represent the mean edge number at which threshold is set; a $\mathrm{NI}-\mathrm{CN}$ s edge number histogram, $\mathbf{b} \mathrm{NI}-\mathrm{CN}$ s edge number boxplot, $\mathbf{c}$ REC-CN edge number histogram, $\mathbf{d}$ REC-CN edge number boxplot

quantified at $r$-values from 1 to 0 at decrements of 0.1 with varying $p$-values (Fig. 3a and c). For the second cycle the edge numbers for the NI-CN were quantified at $r$-values ranging from 0.3 to 0.2 and for the REC-CN at at $r$-values ranging from 0.5 to 0.4 with varying $p$-values (Fig. $3 \mathrm{~b}$ and d). For both latter analyses decrements of 0.01 were used. The boxplot of the NI-CNs illustrates an increased range of edge number the lower the $r$-value became, revealing an increased range from values 0.4 downwards (Fig. 3a). At an $r$-value of 0.3 the complete range for all $100 \mathrm{NI}$-CNs was calculated with 620 to 730 (range $=110$ ) edges present in the networks. At an $r$-value of 0.2 the minimum edge number was estimated with 858 , while the maximum edge number was 1,010, showing an increased range (152). At an $r$-value of 0.23 the complete range for all 100 NI-CNs was calculated with 797 to 905 (range 108) edges present in the networks (Fig. 3b). For the REC-CN an increased range was specifically visible after the correlation coefficient dropped beneath 0.5, i.e. at Pearson's $r=0.5$ the range of edge numbers in all 100 REC-CNs was $58(\min =217, \max =275)$, while at $r=0.4$ the range increased to $98(\min =355, \max =453-$ Fig. 3c). We further investigated $r$-values in-between 0.5 to 0.4 (Fig. 3d), demonstrating a steady increase of median edge numbers the lower the correlation coefficient became. At the targeted $r$-value of 0.41 the minimum edge numbers was estimated with 337 and the maximum edge number 

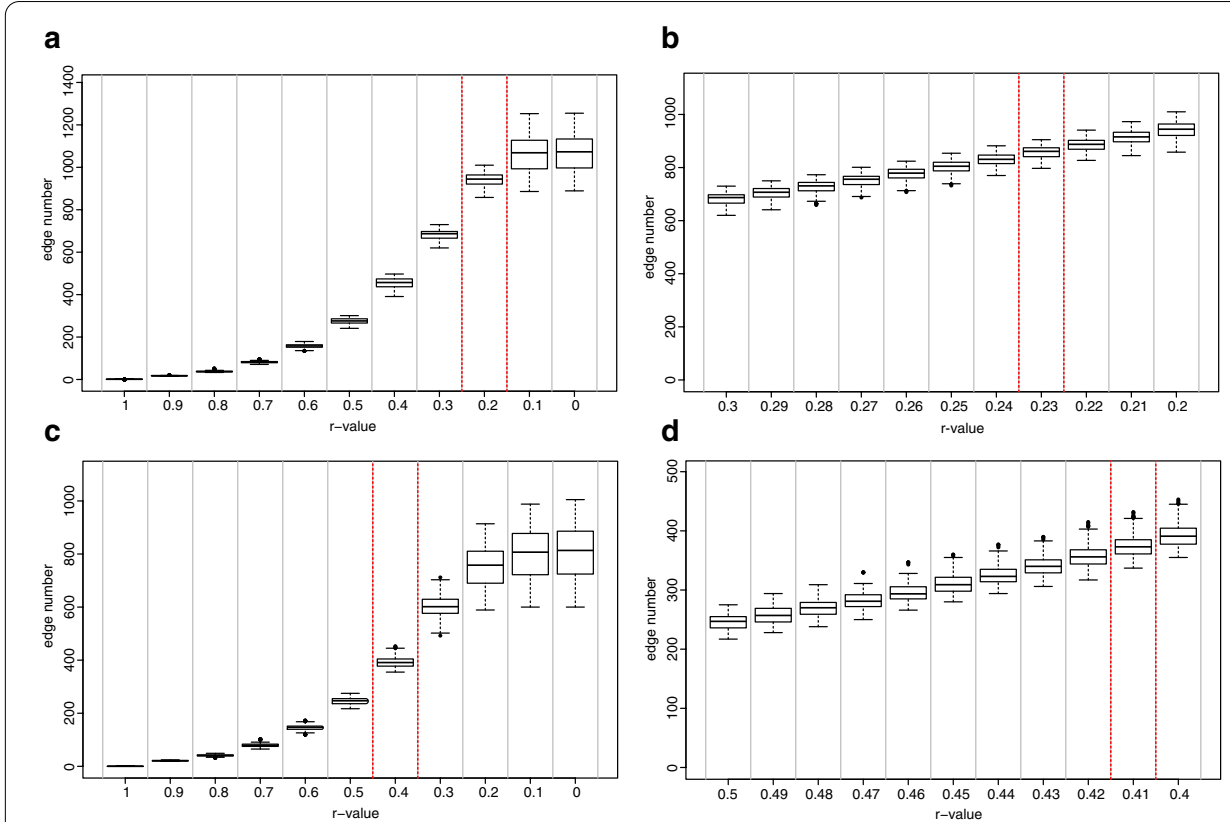

Fig. 3 Edge number distribution of bootstrapped NI and REC networks, $r=1$ to 0 . a NI-CNs edge number boxplot from bootstrapping analysis at different $r$-values, at $r=1$ to 0 at decrements of 0.1 . $\mathbf{b} ~ \mathrm{NI}$-CNs edge number boxplot from bootstrapping analysis at different $r$-values, at $r=0.3$ to 0.2 at decrements of 0.01 . C REC-CNs edge number boxplot from bootstrapping analysis at different $r$-values, at $r=1$ to 0 at decrements of 0.1. d REC-CNs edge number boxplot from bootstrapping analysis at different $r$-values, at $r=0.5$ to 0.4 at decrements of 0.01 . In the boxplots, centerlines represent the median; box limits represent upper and lower quartiles; whiskers represent $1.5 \times$ interquartile range. Bootstrapping was run 100 times with $80 \%$ of the samples allowing replacement. Grey vertical lines separate correlation coefficients, red dashed vertical lines represent proposed correlation coefficient threshold interval

with 421 (range 84). Furthermore, the boxplot revealed increased CIs, indicative for greater standard errors attributed to the increased edge numbers at lower correlation coefficients. These findings underscored the original presupposition of a correlation coefficient threshold setting of Pearson's $|r| \geq 0.23$ in the NI-CN and Pearson's $|r| \geq 0.41$ in the REC-CN.

Next, we used the bootstrapped CNs and computed the CI for each correlation coefficient at varying $p$-values employing the modified Cox test (Fig. 4). For the first cycle, $1 \%$ (empirical $p=0.99$ ) of all NI-CNs at an $r$-value of 0.4 (Fig. 5a) revealed minimum or maximum edge numbers beyond their estimated CI (Fig. 4a); at $r=0.3$, the number rose to $54 \%$, equivalent to an empirical $p$-value of 0.46 . At $r=0.2$, all networks showed to have minimum or maximum edge numbers beyond their estimated CI (empirical $p<0.01$ ). During the second cycle we inspected in particular the targeted $r$-value of 0.23 , revealing that $98 \%$ (empirical p-value 0.02 ) of all networks had minimum or maximum edge numbers beyond their estimated CI (Fig. 4b) For the bootstrapped REC-CNs, significant changing edge numbers as suggested by the CI started to occur at $r=0.5$ (Fig. 4c). Here, 21\% of the CNs showed significant changes (empirical $p=0.79$ ); at $r=0.4,93 \%$ of all REC-CNs exerted significant changes (empirical $p=0.07$ ), and at $r=0.3$, all networks (empirical $p<0.01$ ) had significantly changing edge numbers. For the second cycle we investigated the number of bootstrapped $\mathrm{CNs}$ 


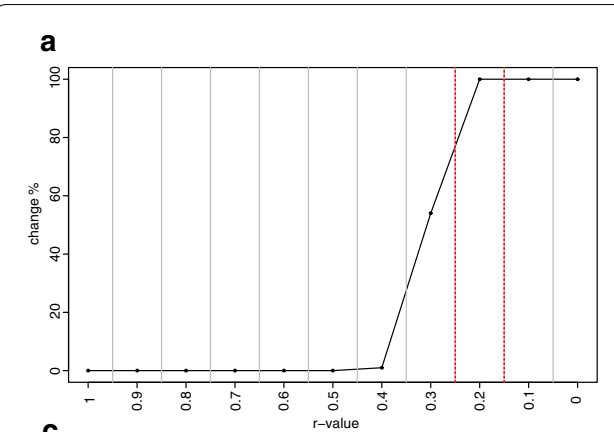

\section{b}

c
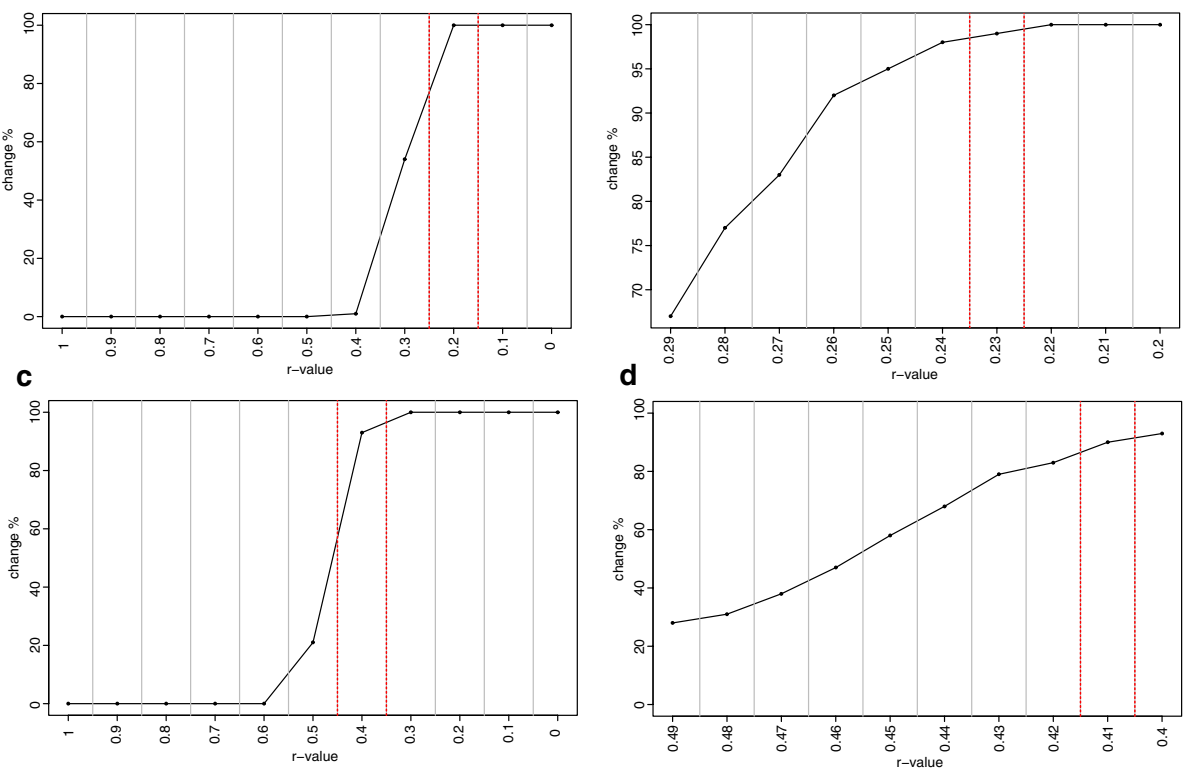

Fig. 4 Relative number of significantly changing bootstrapped NI and REC-CNs. The figure illustrates the relative number of significantly changing edge numbers in bootstrapped $\mathrm{CNs}$. a NI-CNs relative change in edge number based on bootstrapping analysis at different $r$-values, at $r=1$ to 0 at decrements of 0.1 . $\mathbf{b}$ $\mathrm{NI}$ - relative change in edge number based on bootstrapping analysis bootstrapping analysis at different $r$-values, at $r=0.3$ to 0.2 at decrements of 0.01 . c REC-CNs relative change in edge number based on bootstrapping analysis at different $r$-values, at $r=1$ to 0 at decrements of 0.1 d REC-CNs relative change in edge number based on bootstrapping analysis at different $r$-values, at $r=0.5$ to 0.4 at decrements of 0.01 . The confidence interval was estimated by the modified Cox test at different $r$-value to $p$-value combinations (see main text for details). Bootstrapping was run 100 times with $80 \%$ of the samples allowing replacement. Grey vertical lines separate correlation coefficients, red dashed vertical lines represent proposed correlation coefficient threshold interval. a Bootstrapped NI-CNs at $r=1$ to 0 at decrements of $0.1 ; \mathbf{b}$ Bootstrapped REC-CNs at $r=1$ to 0 at decrements of 0.1 ; $\mathbf{c}$ Bootstrapped REC-CNs at $r=0.5$ to 0.4 at decrements of 0.01

associated with the proposed cutoff $r$-value of 0.41 . At this value, $90 \%$ of all networks revealed to have significantly changing edge numbers, which equated to an empirical $p$-value of 0.1 . By setting the $p$-value cutoff threshold to $\leq 0.1$ the bootstrapping analysis confirmed the proposed NI-CN and REC-CN correlation coefficient threshold.

\section{Method validation on mouse metabolite dataset}

To validate the proposed method on a dataset of different biological background, we utilized the mouse metabolite datasets from Batushansky et al. [10]. There the authors tested the effect of fasting in the hearts of mice. For one of the analyses used in the study, the authors constructed CNs for two conditions, i.e. fed and fasted mice. To highlight the differences between networks intersection edges were identified. Networks were constructed at an absolute $r$-value threshold of $\geq 0.7$. Here, we used the datasets for fed and fasted mice and ran it through our correlation-coefficient threshold pipeline. For the first cycle, our method suggested that the correlation coefficient threshold ought to be located in between $r$-values 0.8 and 0.9 for both networks (Fig. 5). For the second cycle, the $r$-value for which the edge number was located within the lower and upper CI was determined with 0.84 for both networks (Fig. 6). At these settings, the Mouse $\mathrm{Fed}^{-\mathrm{CN}}$ 

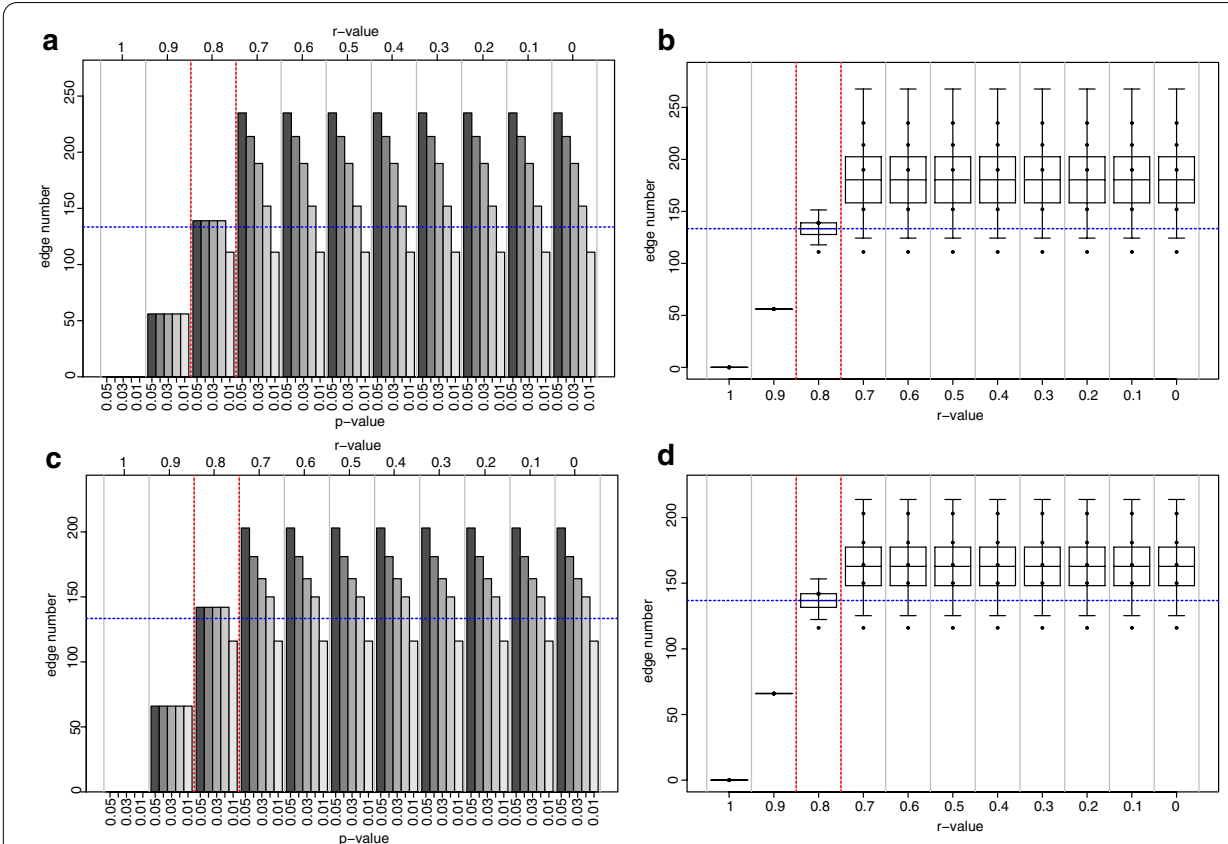

Fig. 5 Edge number distribution of Mouse $_{\text {fed }}$ and Mouse $_{\text {fasted }}$ networks, $r=1$ to 0 . Graphs on the left-hand

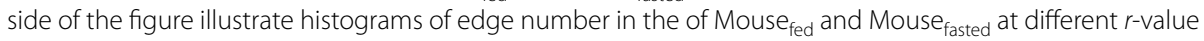
to $p$-value combinations, at $r=1$ to 0 at decrements of 0.1 . Graphs on the right-hand side of the figure depict corresponding boxplots, where the centerlines represent the mean; box limits represent upper and lower standard error; whiskers represent 95\% confidence intervals calculated by the modified Cox test. Grey vertical lines separate correlation coefficients, red dashed vertical lines represent proposed correlation coefficient threshold interval, blue horizontal lines represent the mean edge number at which threshold is set; $\mathbf{a}$ Mouse $_{\mathrm{fed}}-\mathrm{CNs}$ edge number histogram, $\mathbf{b}$ Mouse $_{\mathrm{fed}}-\mathrm{CNs}$ edge number boxplot, $\mathbf{c}$ Mouse $_{\text {fasted }}-\mathrm{CN}$ edge number histogram, $\mathbf{d}$ Mouse $_{\text {fasted }^{-}}$-CN edge number boxplot

had $\left|V_{F e d}\right|=42$ nodes and $\left|E_{F e d}\right|=105$ edges connecting them; the Mouse Fasted CN was composed of $\left|V_{\text {Fasted }}\right|=42$ nodes and $\left|E_{\text {Fasted }}\right|=112$ edges. In the original study the authors identified eight edges intersecting both networks. Here, we identified 17 intersecting edges, containing all edges of the original study (Additional file 1: Supplementary Data S1).

\section{Discussion}

The construction of metabolite $\mathrm{CNs}$ is a non-trivial undertaking. In contrast to weighted gene co-expression networks [20], where all edges are kept within the network, the aim of metabolite CNs is to eliminate some of the correlations [21]. As such, if the correlation coefficient threshold is set too high, valuable biological data may be lost, while if the correlation coefficient threshold is set too low the plethora of edges may have a confounding effect, rendering it difficult to identify the most viable biological information associated with the data at hand. It is therefore highly important to set the correlation coefficient threshold appropriately so that meaningful biological conclusions can be derived. However, the correlation coefficient threshold for metabolite CNs cannot be universally set. Instead, it must be determined dynamically in accordance to the studied system. Although different studies have already applied different correlation coefficient thresholds to construct metabolite CNs, e.g. [1, 4, 

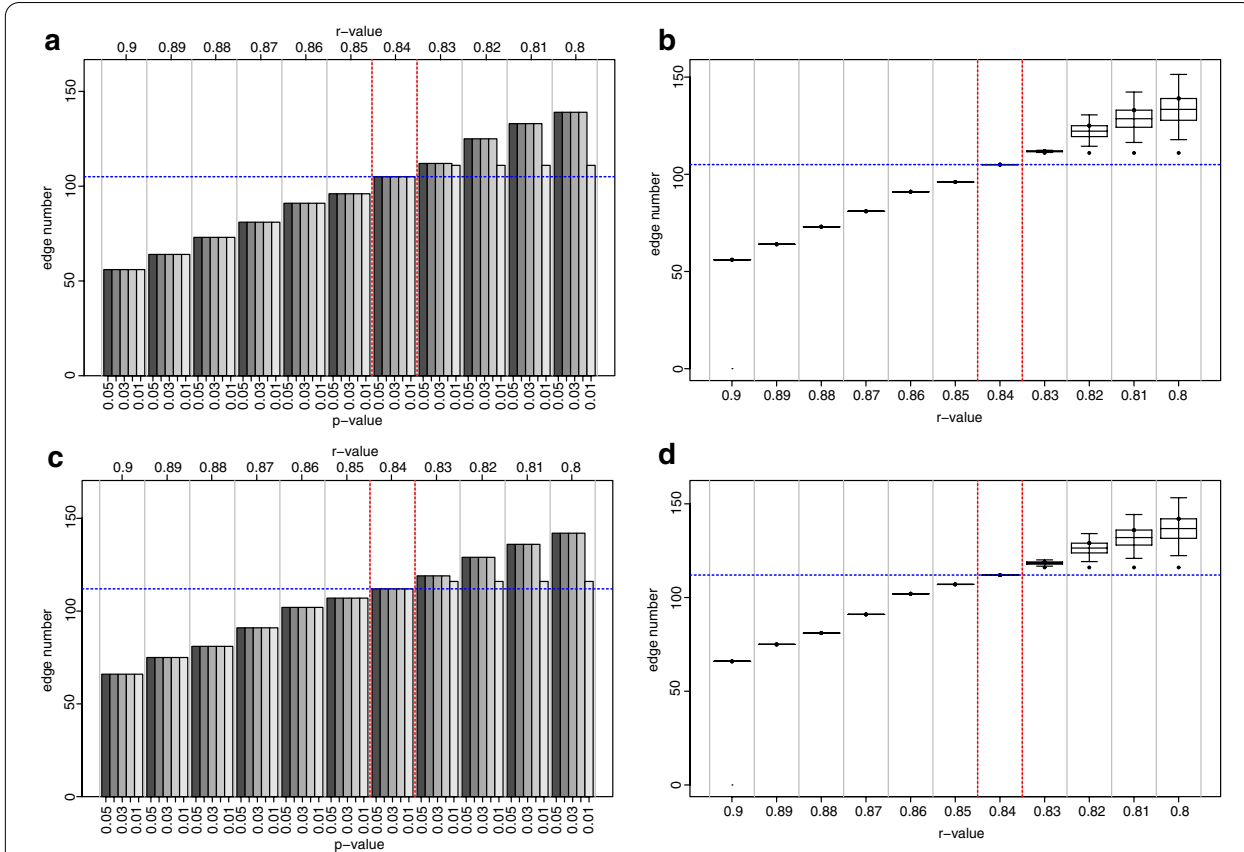

Fig. 6 Edge number distribution of Mouse $_{\text {fed }}$ and Mouse fasted $_{\text {networks, }} r=0.9$ to 0.8. Graphs on the left-hand side of the figure illustrate histograms of edge number in the Mouse $_{\text {fed }}$ and Mouse $_{\text {fasted }}$ at different $r$-value to $p$-value combinations, at $r=0.9$ to 0.8 at decrements of 0.01 . Graphs on the right-hand side of the figure depict corresponding boxplots, where the centerlines represent the mean; box limits represent upper and lower standard error; whiskers represent 95\% confidence intervals calculated by the modified Cox test. Grey vertical lines separate correlation coefficients, red dashed vertical lines represent proposed correlation coefficient threshold interval, blue horizontal lines represent the mean edge number at which threshold is set; a Mouse $_{\text {fed }}-\mathrm{CNs}$ edge number histogram, $\mathbf{b}$ Mouse $_{\text {fed }}-\mathrm{CNs}$ edge number boxplot, $\mathbf{c}$ Mouse $_{\text {fasted- }}$ CN edge number histogram, $\mathbf{d}$ Mouse $_{\text {fasted }^{-}}$-CN edge number boxplot

8-11, 22-24], a set of rules on how to determine them has not been established, yet. Here we introduced an approach that allows researchers to select a correlation coefficient threshold suitable to their studied system.

In our recent study on a potato association panel we constructed a $\mathrm{CN}$ on tuber metabolites and plant morphological traits, where we applied a correlation coefficient threshold of 0.4 [12]. At this setting we identified a critical connection between fumaric acid and plant vigor, which enabled us to identify essential genes aiding the plant to cope with abiotic stresses. Using the same potato dataset, in the current study, we demonstrated how to select the adequate correlation coefficient threshold based on an iterative approach, during which the network topology and specifically its associated edge number was monitored. A series of $\mathrm{CNs}$ were constructed, where different absolute correlation coefficients (from 0 to 1 ) were combined with a set of different $p$-values. To determine the $r$-value threshold a significant change had to be detected. We defined a significant change as the excess of the range of edge numbers beyond the confines of the corresponding $\mathrm{CI}$ of a $\mathrm{CN}$. Once this criterion was fulfilled, the $r$-value threshold was set.

The initial analysis with increments of 0.1 between $r$-values from 0 to 1 suggested a threshold 0.2 for the NI-CN and a threshold 0.4 for the REC-CN (Fig. 1). The second cycle of our proposed method suggested an $r$-value threshold of 0.23 for the NI-CN 
and 0.41 for the REC-CN (Figs. 1 and 2). Bootstrapping analysis with $100 \mathrm{CNs}$ based on $80 \%$ of the samples confirmed the proposed thresholds (Figs. 3 and 4). At these threshold settings the crucial connection between fumaric acid and plant vigor was still present in the REC-CN but not in the NI-CN as suggested in [12]. To validate the proposed method, it was also applied to heart metabolite datasets of fed and fasted mice [10]. The original study proposed a threshold of 0.7 for both CNs. To compare the CNs a network intersection was generated. Our analysis suggested a threshold 0.84 for both CNs. Although our proposed threshold was significantly higher than in the original study, we showed a similar edge intersection.

The proposed thresholds for the four different CNs stretched over a wide range of $r$-values, starting at a correlation coefficient as low as 0.23 for the NI-CN and reaching a correlation coefficient as high as 0.84 for the two mouse CNs. To identify a property that could potentially be key to this finding, we computed a number of network properties for each CN. Network properties derived from node degree (e.g. average degree, edge to node ratio, density, clustering coefficient) varied as much as the proposed correlation coefficient thresholds themselves. However, two other network properties provided interesting results that could potentially be the key elements for correlation coefficient threshold detection, namely: i) the network diameter, which is the maximum geodesic distance between any pair of nodes in a graph; and ii) the network assortativity coefficient, which is the correlation coefficient of degree between pairs of connected nodes [25]. It tells in a concise fashion how nodes are preferentially connected to each other. The diameter for the NI-CN was computed with 6 and its assortativity coefficient with 0.45 , for the REC-CN the diameter was 9 and its assortativity coefficient $=0.36$, for the Mouse $_{\mathrm{Fed}}-\mathrm{CN}$ the diameter was 9 and its assortativity coefficient $=0.47$, and for the Mouse $_{\text {Fasted }}$ CN the diameter was calculated with 8 and its assortativity coefficient with 0.52. Despite the different topologies of the four CNs these two network properties revealed comparable values. We believe that this finding should be further investigated.

\section{Conclusions}

We demonstrated that the approach developed in this study is a valuable tool for the determination of the correlation coefficient threshold for the construction of metabolite CNs. We applied our method to metabolite datasets of different biological background and the thresholds suggested varied from 0.23 over 0.41 to 0.84 . Although the newly proposed $r$-values differed from the values utilized in the original studies, it still allowed us to obtain the same biological conclusions. It is therefore that the network topology of $\mathrm{CN}$ determines the biological interpretation, rather than the strength of the correlation coefficient itself. For this reason, we suggest treating CNs as unweighted graphs once the correlation coefficient has been established and non-significant correlations have been removed.

\section{Methods}

\section{Datasets acquisition and processing}

Datasets for metabolites and morphological traits were adopted from [12]. Preprocessing and quantification of metabolites and morphological traits were performed as described therein. 


\section{CN settings}

The generation of the network was based on the correlation analysis of all metabolites and morphological traits. The Pearson correlation was chosen to estimate correlation coefficients. To construct the initial NI and REC networks, the correlation coefficient threshold was set to 0.4 as previously described. The corresponding $p$-value 0.05 was adjusted via multiple hypotheses testing correction. The mice CNs were constructed as described in [10].

\section{Confidence Interval estimation}

The estimation of the $\mathrm{CI}$ is based on a normal distribution. As the data in this study violated this assumption we employed the CI estimation based on the modified Cox method, which log-transforms the data prior to estimation [19]. It also applies $t$-values rather than $z$-values.

\section{Bootstrapping}

To statistically verify the approach presented in the current study for correlation coefficient threshold settings, we employed bootstrapping with random sample replacement. Bootstrapping was performed 100 times with $80 \%$ of the samples available in the NI and REC datasets.

\section{Availability and requirements}

- Project name: Correlation coefficient threshold determination in correlationbased networks

- Project home page: https://github.com/toubiana/correlation_coefficient_thres hold

- Operating system(s): Platform independent

- Programming language: $\mathrm{R}$

- Other requirements: The source code operates in $\mathrm{R}$ without any dependencies.

- License: ACADEMIC PUBLIC LICENSE

- Any restrictions to use by non-academics: e.g. licence needed

\section{Abbreviations}

$\mathrm{Cl}$ : confidence interval; CNA: correlation-based network analysis; CN: correlation networks; FDR: false discovery rate; GWAS: genome wide association study; NI: normal irrigation; REC: recovery.

\section{Supplementary Information}

The online version contains supplementary material available at https://doi.org/10.1186/s12859-021-03994-z.

Additional file 1. Network intersection. File description: Network intersections of mouse CNs as identified in the current study and in Batushansky et al. [10]. 


\section{Acknowledgements}

We thank Dr. Alfredo Poirier from the Departamento de Ciencias—Matemáticas at PUCP, for useful comments. DT is indebted to Cienciactiva-CONCYTEC and the Instituto Interamericano de Cooperación para la Agricultura (IICA) for supporting his stay at CERMN-PUCP.

\section{Authors' contributions}

DT has designed the study and performed all analyses. Furthermore, DT has written the source code and the manuscript. HM assisted in writing the manuscript. All authors have read and approved the manuscript.

\section{Funding}

This study was funded by the Programa Atracción de Investigadores Cienciactiva-CONCYTEC (008-2017-FONDECYT). The funding body did not contribute to the study design, nor to the collection of samples, analysis, interpretation of data and not in writing of the manuscript.

\section{Availability of data and materials}

All data and material associated with the current study can be found in the supplementary data of [12].

\section{Ethics approval and consent to participate}

Not applicable.

\section{Consent for publication}

Not applicable.

Competing interests

The authors declare that no competing interests exit.

Received: 3 July 2020 Accepted: 2 February 2021

Published online: 10 March 2021

\section{References}

1. Rosental L, Perelman A, Nevo N, Toubiana D, Samani T, Batushansky A, Sikron N, Saranga Y, Fait A: Environmental and genetic effects on tomato seed metabolic balance and its association with germination vigor. BMC Genom 2016, 17.

2. Toubiana D, Puzis R, Wen LL, Sikron N, Kurmanbayeva A, Soltabayeva A, Wilhelmi MDR, Sade N, Fait A, Sagi M et al: Combined network analysis and machine learning allows the prediction of metabolic pathways from tomato metabolomics data. Commun Biol 2019,2

3. Carlin S, Vrhovsek U, Franceschi P, Lotti C, Bontempo L, Camin F, Toubiana D, Zottele F, Toller G, Fait A, et al. Regional features of northern Italian sparkling wines, identified using solid-phase micro extraction and comprehensive twodimensional gas chromatography coupled with time-of-flight mass spectrometry. Food Chem. 2016;208:68-80.

4. Sulpice R, Trenkamp S, Steinfath M, Usadel B, Gibon Y, Witucka-Wall H, Pyl ET, Tschoep H, Steinhauser MC, Guenther $\mathrm{M}$, et al. Network analysis of enzyme activities and metabolite levels and their relationship to biomass in a large panel of Arabidopsis accessions. Plant Cell. 2010;22(8):2872-93.

5. Toubiana D, Xue WT, Zhang NY, Kremling K, Gur A, Pilosof S, Gibon Y, Stitt M, Buckler ES, Fernie AR et al: Correlationbased network analysis of metabolite and enzyme profiles reveals a role of citrate biosynthesis in modulating $\mathrm{N}$ and C Metabolism in Zea mays. Front Plant Sci 2016, 7.

6. Benjamini Y, Hochberg Y. Controlling the false discovery rate - a practical and powerful approach to multiple testing J R Stat Soc B Met. 1995:57(1):289-300

7. Müller-Linow M, Weckwerth W, Hütt MT. Consistency analysis of metabolic correlation networks. BMC Syst Biol. 2007;1:44.

8. Hu T, Zhang W, Fan Z, Sun G, Likhodi S, Randell E, Zhai Y. Metabolomics differential correlation network analysis of Osteoarthritis. Pac Symp Biocomput. 2016;21:120-31.

9. Angelovici R, Batushansky A, Deason N, Gonzalez-Jorge S, Gore MA, Fait A, DellaPenna D. Network-Guided GWAS improves identification of genes affecting free amino acids. Plant Physiol. 2017;173(1):872-86.

10. Batushansky A, Matsuzaki S, Newhardt MF, West MS, Griffin TM, Humphries KM. GC-MS metabolic profiling reveals fructose-2,6-bisphosphate regulates branched chain amino acid metabolism in the heart during fasting. Metabolomics. 2019;15(2):1-12

11. Toubiana D, Sade N, Lifeng L, Wilhelmi MDR, Brotman Y, Luzarowska U, Vogel JP, Blumwald E. Correlation-based network analysis combined with machine learning techniques highlight the role of the GABA shunt in Brachypodium sylvaticum freezing tolerance. Sci Rep. 2020;10(1):1-16.

12. Toubiana D, Cabrera R, Salas E, Maccera C, Franco dos Santos G, Cevallos D, Lindqvist-Kreuze H, Lopez JM, Maruenda H. Morphological and metabolic profiling of a tropical adapted potato association panel subjected to a water recovery treatment reveals new insights into plant vigor. Plant J. 2020;103(6):2193-210.

13. Bae H, Kim SK, Cho SK, Kang BG, Kim WT. Overexpression of OsRDCP1, a rice RING domain-containing E3 ubiquitin ligase, increased tolerance to drought stress in rice (Oryza sativa L.). Plant Sci. 2011;180(6):775-82.

14. Brugiere N, Zhang WJ, Xu QZ, Scolaro EJ, Lu C, Kahsay RY, Kise R, Trecker L, Williams RW, Hakimi S, et al. Overexpression of RING Domain E3 Ligase ZmXerico1 confers drought tolerance through regulation of ABA homeostasis. Plant Physiol. 2017:175(3):1350-69.

15. Joazeiro CAP, Weissman AM. RING finger proteins: mediators of ubiquitin ligase activity. Cell. 2000;102(5):549-52.

16. Mittler R, Kim Y, Song LH, Coutu J, Coutu A, Ciftci-Yilmaz S, Lee H, Stevenson B, Zhu JK. Gain- and loss-of-function mutations in Zat10 enhance the tolerance of plants to ablotic stress. Febs Lett. 2006;580(28-29):6537-42. 
17. Zang DD, Li HY, Xu HY, Zhang WH, Zhang YM, Shi XX, Wang YC: An arabidopsis zinc finger protein increases abiotic stress tolerance by regulating sodium and potassium homeostasis, reactive oxygen species scavenging and osmotic potential. Front. Plant Sci. 2016, 7.

18. Zhang DY, Tong JF, Xu ZL, Wei PP, Xu L, Wan Q, Huang YH, He XL, Yang JY, Shao HB et al. Soybean C2H2-Type zinc finger protein GmZFP3 with conserved QALGGH motif negatively regulates drought responses in transgenic Arabidopsis. Front. Plant Sci 2016, 7.

19. Olsson U. Confidence intervals for the mean of a log-normal distribution. J Stat Edu. 2005;13:1.

20. Langfelder P, Horvath S. WGCNA: an R package for weighted correlation network analysis. BMC Bioinform. 2008;29(9):559.

21. Toubiana D, Fernie AR, Nikoloski Z, Fait A. Network analysis: tackling complex data to study plant metabolism. Trends Biotechnol. 2013;31(1):29-36.

22. Hochberg U, Degu A, Toubiana D, Gendler T, Nikoloski Z, Rachmilevitch S, Fait A: Metabolite profiling and network analysis reveal coordinated changes in grapevine water stress response. BMC Plant Biol. 2013, 13(84).

23. Lisec J, Romisch-Margl L, Nikoloski Z, Piepho HP, Giavalisco P, Selbig J, Gierl A, Willmitzer L. Corn hybrids display lower metabolite variability and complex metabolite inheritance patterns. Plant J. 2011;68(2):326-36.

24. Toubiana D, Semel Y, Tohge T, Beleggia R, Cattivelli L, Rosental L, Nikoloski Z, Zamir D, Fernie AR, Fait A: Metabolic profiling of a mapping population exposes new insights in the regulation of seed metabolism and seed, fruit, and plant relations. Plos Genet. 2012, 8(3)

25. Newman, MEJ: Assortative mixing in networks. Phys. Rev. Lett. 2002, 89(20)

\section{Publisher's Note}

Springer Nature remains neutral with regard to jurisdictional claims in published maps and institutional affiliations.

- fast, convenient online submission

- thorough peer review by experienced researchers in your field

- rapid publication on acceptance

- support for research data, including large and complex data types

- gold Open Access which fosters wider collaboration and increased citations

- maximum visibility for your research: over 100M website views per year

At BMC, research is always in progress.

Learn more biomedcentral.com/submissions 